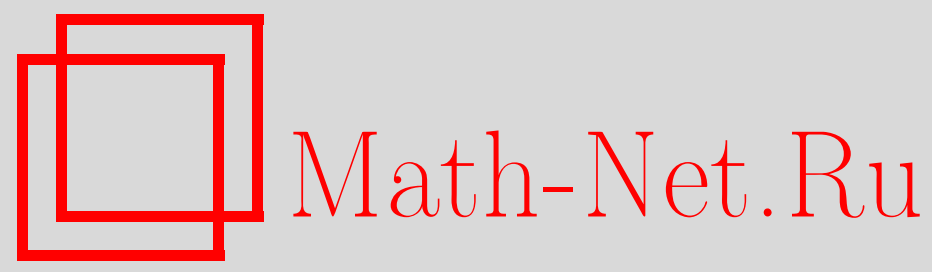

А. И. Созутов, О группах с конечной инволюцией и локально конечной 2-изолированной подгруппой четного периода, Матем. заметки, 2001, том 69, выпуск 6, 912-918

DOI: https://doi.org/10.4213/mzm705

Использование Общероссийского математического портала Math-Net.Ru подразумевает, что вы прочитали и согласны с пользовательским соглашением http://www . mathnet.ru/rus/agreement

Параметры загрузки:

IP : 54.89 .56 .158

26 апреля 2023 г., 18:15:57

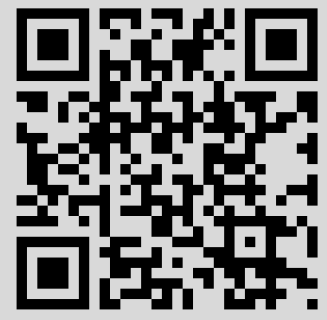




\section{О ГРУППАХ С КОНЕЧНОЙ ИНВОЛЮЦИЕЙ И ЛОКАЛЬНО КОНЕЧНОЙ 2-ИЗОЛИРОВАННОЙ ПОДГРУППОЙ ЧЕТНОГО ПЕРИОДА}

\section{А. И. Созутов}

Собственная подгруппа $H$ группы $G$ назьвается сильно изолированной, если она содержит централизатор каждого своего неединичного элемента, и 2-изолированной, если из условий $C_{G}(g) \cap H \neq 1$ и $2 \in \pi\left(C_{G}(g)\right)$ следует $C_{G}(g) \leqslant H$. Инволюция $i$ группы $G$ называется конечной, если $\left|i i^{g}\right|<\infty(\forall g \in G)$. В работе исследуется группа $G$ с конечной инволюцией $i$ и 2 -изолированной локально конечной подгруппой $H$, содержащей инволюцию. Установлено, что выполняется хотя бы одно из следующих утверждений:

1) все 2-элементы группы $G$ содержатся в $H$;

2) $(G, H)$ - пара Фробениуса, $H$ совпадает с централизатором своей единственной инволюции и все инволюции в $G$ сопряжены;

3) $G=F \lambda C_{G}(i)$ - локально конечная группа Фробениуса с абелевым ядром $F$;

4) $H=V \lambda D$ - группа Фробениуса с локально цик лическим неинвариантным множителем $D$ и сильно изолированным ядром $V, U=O_{2}(V)$ - силовская 2-подгруппа группы $G$ и $G-Z$-группа подстановок множества $\Omega=\left\{U^{g} \mid g \in G\right\}$.

Библиография: 13 названий.

Собственная подгруппа $H$ групшы $G$ называется сильно изолированной, если она содержит централизатор каждого своего неединичного элемента, т.е. для любого неединичного элемента $g \in G$ из условия $C_{G}(g) \cap H \neq 1$ следует $C_{G}(g) \leqslant H$, и 2-изолированной, если это условие выполняется для $C_{G}(g)$, содержаших инволюции [1]. Инволюция $i$ групшы $G$ назьвается конечной, если $\left|i i^{g}\right|<\infty(\forall g \in G)$ [2]. Группу $G$ называем группой четного периода (нечетного периода), если она содержит инволюцию (не содержит инволюций). Собственная подгруппа $H$ группы $G$ назьвается сильно вложенной, если $2 \in \pi(H)$ и $2 \notin \pi\left(H \cap H^{g}\right)(\forall g \in G \backslash H)$ [3].

Конечные групш с сильно изолированными подгруппами изучены в 60-х годах [4]-[7]. Так М. Судзуки [5] и В.М.Бусаркин [6] показали, что конечная группа, содержашая сильно изолированную подгруппу четного поря дка, является либо группой Фробениуса, либо $Z T$-группой, т.е. дважды транзитивной группой подстановок нечетной степени, в которой лиш единица оставляет на месте три различньх точки. В [1] Бусаркин ввел понятие 2 -изолированной подгруппы и доказал, что конечная группа $G$ с 2 -изолированной подгруппй $H$ четного порядка, вне которой есть 2-элементы, имеет то же строение.

Работа выполнена при поддержке Российского фонда фундаментальных исследований, грант № 99-01-00542, и ККФН, грант № 9F132. 
Цель данной заметки - доказательство бесконечного аналога теоремы Бусаркина [1]. Предварительно сделаем несколько замечаний. Как вытекает из теоремы Куроша [8], каждьй множитель свободного произведения $G$ неединичных групा $H_{i}$ сильно изолирован в $G$. Следовательно, для содержательного результата необходимы дополнительные ограничения. Таковыми в теореме являются локальная конечность подгруппы $H$ и существование конечной инволющии в $G$. Для бесконечных групп в общем случае результат Фробениуса неверен (см., например, [9], [10]), поэтому в формулировке теоремы добавилось утверждение 2). Насколько это обосновано, пока неизвестно (см. по этому поводу также вопросы $4.74,10.64,11.52,12.48$ из Коуровской тетради [11]). Поскольку для бесконечных групп понятие четности или нечетности степени групшы подстановок не имеет смысла, изменена соответствующая часть теоремы (утверждение 4)). Кроме того, одно из условий теоремы из [1] оказалось удобней сформулировать в качестве утверждения 1).

ТЕОРемА. Пусть группа $G$ содержит 2-изолированную локально конечную подгруппу $Н$ четного периода и конечную инволючию $i$. Тогда справедливо хотя бы одно из следующих утверждений:

1) все 2-әлементы группь $G$ содержатся в $H$;

2) $(G, H)$ - пара Фробениуса, $H$ совпадает с иентрализатором своей единственной инволюиии и все инволюиии в $G$ сопряжены;

3) $G=F \lambda C_{G}(i)$ - локально конечная группа Фробениуса с абелевым ядром $F$;

4) $H=V \lambda D$ - әруппа Фробениуса с локально ииклическим неинвариантным множителем $D$ и сильно изолированным ядром $V, U=O_{2}(V)-c u$ ловская 2-подгруппа группы $G$ и $G$ - Z-группа подстановок множества $\Omega=\left\{U^{g} \mid g \in G\right\}$.

\section{1. Случай 2-ранга 1.}

Лемма 1 [1]. Справедливы следующие свойства:

1) пересечение любого мнохсества 2-изолированных подгрупп в произвольной группе является 2-изолированной подгруппой;

2) подгруппа, сопряженная 2-изолированной подгруппе, 2-изолирована;

3) если $A$ 2-изолирована в $B$, a $B$ 2-изолирована в $C$, то $A$ 2-изолирована ${ }_{\boldsymbol{B}} C$

4) если 2-изолированная подгруппа $H$ группы $G$ имеет четный порядок, то $H$ содержит каждую силовскую 2-подгруппу группь $G$, нетривиально пересекаюшучося с $\mathrm{H}$.

Далее предполагается, что группа $G$ удовлетворяет условиям теоремы и не все инволюции из $G$ содержатся в $H$.

ЛЕМма 2. Если в $G \backslash H$ есть инволюиия, то все инволюиии в $G$ сопряжены. $B$ частности, любая инволючия в $G$ конечна.

ДоКАЗАТЕЛЬСТВО. Достаточно показать сопряженность двух инволюций $u$ и $v$, взятых из $H$ и $G \backslash H$ соответственно. Предположим вначале, что хотя бы одна из этих инволюций конечна. Рассмотрим $K=\langle u, v\rangle$. Очевидно, подгруппа $K$ конечна, не содержится в $H$ и имеет с $H$ нетривиальное пересечение. Поэтому $Z(K)=1$ и $K$-группа 
Фробениуса. Это влечет сопряженность $u$ и $v$ в $K$. Отсюда заключаем, что $H$ содержит конечную инволюцию. Поэтому можно считать, что инволюция $u$ конечна, а $v-$ произвольная инволюция из $G \backslash H$. По доказанному вьше $u$ и $v$ сопряжены. Лемма доказана.

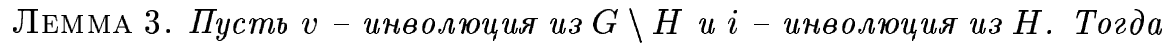

1) $D=H \cap H^{v}$ - периодическая абелева группа $u$ v инвертирует $D$;

2) множества инволюиий из $i^{H}$, әлементов из $D$ и инволючий правого смежного класса Нv равномощны; при этом если $i$ - фиксированная инволючия из $H$, то каждой инволючии $k \in i^{H}$ (в том числе и инволюиии $i$ ) соответствует единственная инволючия $j_{k} \in H v$ такая, что viv $=j_{k} i j_{k}$;

3) подгруппа $D$ сильно изолирована в $H$ и $F=C_{G}(D)$ - периодическая абелева сильно изолированная в $G$ подгруппа без инволюиий;

4) в каждом смежном классе $C_{H}(i) \cdot b$, где $b \in H$, существует и при том единственный инвертируемый инволюцией $v$ әлемент; в частности, $H=$ $D \cdot C_{H}(i)$

5) если $D=1$, то $H$ совпадает с иентрализатором $C_{G}(i)$ некоторой инволюичи $i$

6) подгруппа $H$ совпадает с иентрализатором $C_{G}(i)$ некоторой инволючии $i$ в том и только том случае, когда $(G, H)$ - пара фробениуса.

ДокаЗАТЕЛЬСТво. 1) Понятно, что $v \in N_{G}(D)$ и $C_{D}(v)=1$. Ввиду леммы 2 инволюция $v$ конечна. Но тогда для любого $h \in D$ имеем $\left|v v^{h}\right|\left\langle\infty,\left\langle v, v^{h}\right\rangle=\langle v, h\rangle\right.$, причем $h^{v}=h^{-1}$, что и доказьвает утверждение.

2) Пусть $i, k, t$ - не обязательно различные инволюции из $i^{H}, V=\langle v k v, i\rangle$ и $W=$ $\langle v k v, k\rangle$. Очевидно, что $v \in N_{G}(W)$, поэтому $v k v \notin H, V$ - конечная группа Фробениуса и в $V$ найдется инволюция $j_{k}$ такая, что $j_{k} i j_{k}=v k v$. Поскольку инволюции $i$ и $k$ сопряжены в $H$, то $j_{k} \in H v$. Аналогично определяется инволющия $j_{t} \in H v$. Если $k \neq t$, то $v k v=j_{k} i j_{k} \neq v t v=j_{t} i j_{t}$ и $j_{k} \neq j_{t}$.

Пусть теперь $j$ - произвольная инволюция из $H v, v=h j$, где $h \in H$. Понятно, что $h=v j \in D$. Положим $k=h i h^{-1}$. Тогда $v k v=j i j, j \in\langle i, v k v\rangle$ и $j=j_{k}$. Таким образом, соответствие $k \leftrightarrow j_{k}$ биективно. Из доказанного также следует, что множество инволюций из $H v$ совпадает с множеством $D v$ и утверждение верно.

3) Покажем вначале, что для любого элемента $b \in D$ подгруппа $C_{G}(b)$ не содержит инволюций. Действительно, если $j \in C_{G}(b)$ - инволюция, то $C_{G}(b) \leqslant H$. Учитывая утверждение 1) леммы, имеем $v \in N_{G}\left(C_{G}(b)\right)$ и $L \cap H-2$-изолированная подгруппа четного периода групшы $L=C_{G}(B) \lambda\langle v\rangle$. Но тогда ввиду леммы 2 инволюции $v$ и $j$ сопряжены в $L$; получили противоречие.

Если бы централизатор некоторого элемента из $M=C_{G}(b)$ имел четньй период, то этот централизатор как содержаший элемент $b$ имел бы неединичное пересечение $c$ 2 -изолированной подгруппй $D$ и поэтому содержался бы целиком в $D$ (лемма 1 ). Это противоречит нечетности периода группы $D$. Значит, централизатор любого неединичного элемента из $M$ не содержит инволюций. Но тогда по тем же соображениям, что и в доказательстве утверждения 1), $M$ - абелева периодическая подгруппа, инвертируемая инволюцией $v, M=C_{G}(d)$ для любого элемента $d \in M$ и подгруппа $F=C_{G}(D)$ совпадает с $C_{G}(b)$ для каждого элемента $b \in F^{\#}$ и сильно изолирована в $G$. Отсюда также следует $H \cap C_{G}(d)=D$ для любого неединичного элемента $d \in D$, поэтому $D$ 
сильно изолирована в $H$. Утверждение доказано.

4) Пусть $b$ - любой элемент из $H$. По утверждению 2) леммы смежньй класс $H v$ содержит единственную инволюцию $j_{k}$ такую, что $(b v)^{-1} i(b v)=j_{k} i j_{k}$. Это означает, что $b v j_{k} \in C_{H}(i), c=v j_{k} \in b^{-1} \cdot C_{H}(i)$ и элемент $j_{k} v \in C_{H}(i) \cdot b$ инвертируется инволюцией $v$. Если $d=t v$ - произвольньй инвертируемый инволюцией $v$ элемент из $C_{H}(i) b$, то $b v t \in C_{H}(i),(b v)^{-1} i(b v)=t i t, t=j_{k}$, по утверждению 2) леммы и $d=c$. Таким образом, $D$ содержит ровно по одному представителю каждого смежного класса $H$ по подгрупше $C_{H}(i)$, значит $H$ факторизуется подгруппами $D$ и $C_{H}(i)$.

5) Поскольку $D=1$, то ввиду утверждения 4) $H=C_{H}(i)$ для произвольной инволющии $i \in H$. Утверждение доказано.

6) Пусть $(G, H)$ - пара Фробениуса. Тогда $D=1$ и по утверждению 5) $i$ - единственная инволюция в $H$. Обратно, если $i$ - единственная инволюция в $H$, то ввиду утверждения 2) $(G, H)$ - пара Фробениуса. Лемма доказана.

Таким образом, если $i$ - единственная инволюция в $H$, то имеет место утверждение 2 ) теоремы (в силу лемм 2,3$)$. Пусть теперь $H$ содержит более одной инволюции. Тогда при условиях леммы 4 справедливо утверждение 3) теоремы.

Лемма 4. Пусть $v$ - инволюиия из $G \backslash H, 1 \neq D=H \cap H^{v} u N_{H}(D) \neq D$. Тогда $F=C_{G}(D)$ - периодическая абелевая нормальная в $G$ подгруппа, каждая инволюиия из $G$ инвертирует $F u G=F \lambda C_{H}(i)$ - локально конечная группа Фробениуса.

ДокАЗАТЕЛЬСтво. В силу условий и утверждения 2) леммы $3 D=H \cap H^{v} \neq 1$ и $N_{H}(D) \neq D$. Ввиду локальной конечности $H$ и сильной изолированности $D$ в $G$ $N_{H}(D)$ - групша Фробениуса с неинвариантным множителем $T$ (см., например, [9]). Пусть $1 \neq t \in T$. Из леммы 3 следует, что $t \in N_{G}(F), v F \in Z\left(N_{G}(D) / F\right)$, поэтому $t \leqslant C_{G}(i)$ для некоторой инволюции $i \in v F$. Но тогда $i \in C_{G}(t) \leqslant H$. Понятно, что $i$ инвертирует $D$ и ввиду леммы $3 i^{H}=i^{D}$. Отсюда выводим, что подгруппа $D$ нормальна в $H$ и инвертируется любой инволюцией из $H$. Теперь легко показать, что множество $i^{D}$ исчерпывает множество всех инволюций из $H$. Таким образом, $H \leqslant N_{G}(D)$. Ввиду локальной конечности $H$ и сильно изолированности $D$ в $H$ заключаем, что $H=D \lambda C_{H}(i)$ - группа $\Phi$ робениуса с неинвариантным множителем $C_{H}(i)$ и ядром $D$.

Пусть $k$ - произвольная инволющия из $G \backslash H$ и $H_{k}=H \cap H^{k}$. По лемме $3 H_{k}$ - периодическая абелева подгруппа группы $H$, действующая при сопряжении транзитивно на множестве $i^{D}$. Из свойств локально конечных групп Фробениуса (см., например, [9]) легко следует, что $H_{k}=D$. Это означает, что инволюция $k \in G$ инвертирует $D$. Ввиду леммы 1 все инволющии из $G$ исчерпывают множество $i^{G}$, при этом $C_{G}(i)=C_{H}(i)$. Поэтому $V=\left\langle i^{G}\right\rangle=C_{V}(D) \lambda\langle i\rangle$. Но тогда $C_{V}(D)=F$ и $F$-периодическая абелевая сильно изолированная подгруппа нечетного периода, инвертируемая каждой инволюцией из $G$ (лемма 3$)$. Следовательно, все инволюции в $V$ сопряжены и $G=F \lambda C_{H}(i)$. Поскольку централизатор любого элемента из $C_{H}(i)$ содержится в $C_{H}(i)$, причем $C_{H}(i)-$ локально конечная группа, то $G=F \lambda C_{H}(i)$ - группа Фробениуса и в этом случае лемма доказана.

2. Случай 2 -ранга $\geqslant 2$. Ввиду лемм 3,4 остается рассмотреть случай, когда $1 \neq D=H \cap H^{v}$ и $N_{H}(D)=D$ для некоторой инволюции $v$ из $G \backslash H$. Покажем, что в этом случае реализуется утверждение 4) теоремы. 
Лемма 5. Пусть $v-$ инволюиия из $G \backslash H, 1 \neq D=H \cap H^{v} u N_{H}(D)=D$. Тогда

1) $H=V \lambda D$ - әруппа Фробениуса с локально ииклическим неинвариантным множителем $D$ и нильпотентным ядром $V$ четного периода;

2) подгруппа $V$ сильно изолирована в $G$;

3) силовская 2-подгруппа $U$ группы $H$ нормальна в $H$;

4) подгруппа $B=N_{G}(U)$ сильно вложсена в $G$.

ДокАЗАТЕЛЬСТво. 1) Поскольку $D$ сильно изолирована в $H, N_{H}(D)=D$ и $H$ локально конечна, то ввиду хорошо известных результатов теории локально конечных групп (Фробениуса) (см., например, [9]) $H=V \lambda D$ - группа Фробениуса с локально циклическим неинвариантным множителем $D$ и нильпотентным ядром $V$ четного периода.

2) Из нильпотентности $V$ и 2-изолированности $H$ следует $C_{G}(f) \leqslant H$ для любого элемента $1 \neq f \in V$ и ввиду утверждения 1) $C_{G}(f) \leqslant V$.

3) Поскольку $U \leqslant V$ и $V$ нильпотентна, то $U$ нормальна в $H$.

4) По условиям леммы не все инволюции из $G$ содержатся в $U$; следовательно, $B=$ $N_{G}(U) \neq G$. Ввиду нильпотентности $V$ и ее сильно изолированности в $G$ для $g \in G \backslash B$ выполняется $V \cap V^{g}=1$ и $B \cap V^{g}=1$. Отсюда $2 \notin \pi\left(B \cap B^{g}\right)$ и лемма доказана.

Доказательства утверждений следующих ниже лемм можно найти в [12], [13], [2].

Лемма 6 [2, лемма 2.1]. Пусть $G$ - әруппа с сильно вложснной подәруппой $B$ и конечной инволюиией. Тогда

1) все инволюиии в $G$ сопряжены и все инволюиии из $B$ сопряжены в $B ; \boldsymbol{\theta}$ частности, любая пара инволючий в $G$ порождает конечную подгруппу;

2) между множеством инволюиий из $B$ и множеством инволюиий любого правого смехсного класса $\mathrm{Bg}$, где $\mathrm{g} \in \mathrm{G}$, мохсно установить взаимно однозначное соответствие; при этом если $i$ - фиксированная инволюиия из $B$, то каждой инволюиии $k \in B$ (в том числе и инволюции $i$ ) соответствует единственная инволюиия $j_{k} \in B g$ такая, что $g^{-1} \mathrm{~kg}=j_{k} i j_{k}$;

3) любой әлемент $g \in G$ обладает представлением $g=h j$, әде $h \in B, a j-$ некоторая инволюиия;

4) для любой инволюции $j \in G \backslash B$ в подгруппе $B$ сущ,ствует множество $M_{j}$ инвертируемых инволюиией $j$ әлементов той же мощности, что и мнохество инволючий из $B$.

ЛЕмма 7 [2, лемма 2.2]. Пусть $G$ - группа с сильно вложенной подгруппой $B u$ конечной инволюиией, $i \in B u j \in G \backslash B-$ произвольные инволючии, $H_{j}=B \cap B^{j} u$ $T_{j}$ - подгруппа в $B$, порожденная всеми инвертируемыми инволюиией $j$ әлементами из В. Тогда

1) $T_{j} \leqslant H_{j}, H_{j}$ - подгруппа без инволючий, $\left\langle j, T_{j}\right\rangle=T_{j} \lambda\langle j\rangle u\left\langle j, H_{j}\right\rangle=H_{j} \lambda\langle j\rangle$;

2) в каждом смежном классе $C_{B}(i) \cdot b$, где $b \in B$, существует единственньй инвертируемый инволюиией $j$ әлемент, и его порядок конечен и нечетен;

3) $B=T_{j} \cdot C_{B}(i)=H_{j} \cdot C_{B}(i)$ и $i^{T_{j}}$ - множество всех инволючий группь $B$;

4) если $b$ - неединичный строго вещественный относительно инволюиии $j \in$ $G \backslash B$ әлемент из $B$, то $C_{B}(b)$ не содержит инволюиий. 
Таким образом, далее $B=N_{G}(U)$ - сильно вложенная подгруппа, $V$ - периодическая нильпотентная сильно изолированная подгруппа 2-ранга $\geqslant 2, U$ - силовская 2 -подгруппа из $V$.

Лемма 8. Пусть $v-$ произвольная инволющия из $G \backslash B u T=B \cap B^{v}$. Справедливы следуюшие утверждения:

1) $B=V \lambda T$ и все инволючии из $U$ содержстся в ее иентре $Z(U)$;

2) $T$ - периодическая локально ииклическая группа, инвертируемая инволюиией $v$ и действующая транзитивно на множсестве всех инволюций из $U$;

3) $B=V \lambda T-$ локально конечная группа Фробениуса с ядром $V$ и локально ииклическим дополнением $T$;

4) подгруппа $T$ сильно изолирована в $G$ и $W=N_{G}(T)=T \lambda C_{W}(v)$ - группа Фробениуса с конечным дополнением $C_{W}(v)$.

ДокАЗАТЕЛЬСТво. 1) По лемме 7 подгруппа $T$ не содержит инволюций и $B=$ $T \cdot C_{B}(i)$, где $i$ - произвольная инволюция из $U$. Поскольку $C_{B}(i) \leqslant V$ и $V$ сильно изолирована, то $B=V \cdot T$ и $C_{B}(i) \geqslant U$. Утверждение доказано.

2) Предположим вначале, что $C=C_{T}(v) \neq 1$. Тогда, очевидно, подгруппа $C$ сопряжена с некоторой подгруппой из $V$. Поскольку $V$ сильно изолирована в $G$ (лемма 3 ) и нормальна в $B$ (лемма 5 ), то $C \leqslant V$, противоречие. Следовательно, $C_{T}(v)=1$ и $T-$ периодическая абелева подгруппа, инвертируемая инволюцией $v$. Теперь леммы 6,7 завершают доказательство утверждения.

3) По лемме $7 T \cap V=1$ и ввиду сильной изолированности $V$ в $G$ и равенства $B=V T$ заключаем, что $B=V \lambda T$ - группа дополнением $T$.

4) Пусть $1 \neq t \in T$ и $x \in C_{G}(t) \backslash T$. Тогда $x \in G \backslash B$ и по лемме $6 x=h j, h \in B$, $j$ - инволюция. Понятно, что $x \in B \cap B^{x}=B \cap B^{j}=K$. По утверждению 3) леммы $K$ - неинвариантньй множитель групшы робениуса $B=V \lambda T$. Поскольку $t \in K$, то $K=T$ и по утверждению 2) леммы $j$ инвертирует $t$. Но тогда $h$ также инвертирует $t$, противоречие. Следовательно, $C_{G}(t)=T$ и подгруппа $T$ сильно изолирована в $G$. Отсюда и из локальной цикличности $T$ выводим, что $T=C_{G}(T)$ имеет конечный индекс в $W=N_{G}(T)$ и $W=T \lambda C_{W}(v)$ - локально конечная группа $\Phi$ робениуса. Лемма доказана.

Обозначим через $\Omega$ множество всех подгрупп из $G$, сопряженных с подгруппой $U$. Далее будем рассматривать подстановочное представление группы $G$ на множестве $\Omega$, при этом обозначим $U=\alpha \in \Omega$. Доказательство теоремы завершает

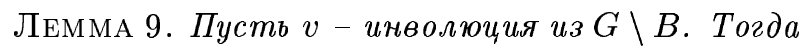

1) $v^{B}$ - мнохсество всех инволюиий из $G \backslash B$;

2) группа $G-2$-транзитивна на $\Omega$, при этом $B=G_{\alpha}-$ стабилизатор точки u $T=G_{\alpha \beta}-$ стабилизатор двух точек;

3) подгруппа $V$ действует регулярно на мнохестве $\Omega \backslash\{\alpha\}$;

4) только единичный әлемент группы $G$ оставляет на месте три различные точки из $\Omega$.

ДокАЗАТЕЛЬСтво. 1) Пусть $T=B \cap B^{v}$. Поскольку по лемме 8 для любой инволюции $j \in G \backslash B$ подгрупша $T_{j}=B \cap B^{j}$ есть неинвариантньй множитель групшы 
$\Phi$ робениуса $B=V \lambda T$, то $T_{j}^{u}=T$ для некоторого $u \in V$, и ввиду последнего утверждения той же леммы инволющии $v$ и $j^{u}$ сопряжены с помощью подходящего элемента $t \in T$. Это и означает, что $j \in v^{B}$. Утверждение доказано.

2) Очевидно, что $B=G_{\alpha}$. Ввиду утверждения 1) $B$ транзитивна на $\Omega \backslash\{\alpha\}$. Следовательно, $G$ - 2-транзитивна на $\Omega$. Из лемм 6-8 вытекает, что для любого $g \in G \backslash B$ пересечение $B \cap B^{g}$ совпадает с подходяшим неинвариантньм множителем $T^{u}$ грушшы $B$, здесь $u \in V$. Последнее равносильно тому, что $T=G_{\alpha \beta}$ - стабилизатор двух точек. Утверждение доказано.

3) Поскольку $G_{\alpha}=V \lambda G_{\alpha \beta}$ и $B$ транзитивна на $\Omega \backslash\{\alpha\}$, то подгруппа $V$, очевидно, регулярна на $\Omega \backslash\{\alpha\}$. Утверждение доказано.

4) Пусть $\gamma \in \Omega \backslash\{\alpha, \beta\}$ и $u \in V$ элемент такой, что $\beta^{u}=\gamma$. Поскольку $T$ - неинвариантньй множитель групшы $\Phi$ робениуса $B$, а $u$ - неединичньй элемент из ее ядра $V$, то $T \cap T^{u}=1$. Следовательно, $G_{\alpha \beta \gamma}=1$ как подгруппа тривиального пересечения $T \cap T^{u}$. Ввиду 2 -транзитивности $G$ заключаем, что ее стабилизатор любых трех точек из $\Omega$ тривиален, т.е. $G-Z$-группа [3]. Лемма и вместе с ней теорема доказаны.

\section{СПИСОК ЦИТИРОВАННОЙ ЛИТЕРАТУРЫ}

[1] Бусаркин В. М. О 2-изолированных подгруппах // Матем. заметки. 1968. Т. 3. №5. C. $497-501$.

[2] Созутов А.И. О некоторых бесконечных группах с сильно вложенной подгруппой // Алгебра и логика (в печати).

[3] Горенстейн Д. Конечные простые группы. М.: Мир, 1985.

[4] Feit W. On groups which contain Frobenius groups as subgroups // Proc. Symp. Pure Math. 1959. P. 22-28.

[5] Suzuki M. Two characteristic properties of $(Z T)$-groups // Osaka Math. J. 1963. V. 15. P. $143-150$.

[6] Бусаркин В.М. Строение изолированных подгрупп в конечных группах // Алгебра и логика. 1965. Т. 4. № 2. С. 33-50.

[7] Herzog M. On finite groups which contain a Frobenius subgroup // J. Algebra. 1967. V. 6. P. $192-221$.

[8] Курош А. Г. Теория групп. М.: Наука, 1967.

[9] Старостин А. И. О группах Фробениуса // Укр. матем. ж. 1971. Т. 23. № 5. С. 629-639.

[10] Созутов А. И., Шунков В. П. Об одном обобщении теоремы Фробениуса на бесконечные группы // Матем. сб. 1976. Т. 100. № 4. С. 495-506.

[11] Коуровская тетрадь. Нерешенные вопросы теории групп. 14-е изд. Новосибирск, 1999.

[12] Шунков В. П. Мp-группы. М.: Наука, 1990.

[13] Шунков В. П. О вложении примарных элементов в группе. Новосибирск: Наука, 1992.

г. Красноярск

Поступило

E-mail : root@sozutov.krasnoyarsk.su 